\title{
The assessment of hemineglect syndrome with cancellation tasks: a comparison between the Bells test and the Apples test
}

\author{
Benedetta Basagni $^{1}$ (i) $\cdot$ Antonio De Tanti $^{1} \cdot$ Alessio Damora $^{2} \cdot$ Laura Abbruzzese $^{2}$ • \\ Valentina Varalta ${ }^{3}$. Gabriella Antonucci ${ }^{4,5}$ - Wai Ling Bickerton ${ }^{6}$. Nicola Smania ${ }^{3}$. \\ Mauro Mancuso ${ }^{2}$
}

Received: 10 July 2017 / Accepted: 26 September 2017 / Published online: 4 October 2017

(C) Springer-Verlag Italia S.r.l. 2017

\begin{abstract}
Unilateral spatial neglect (USN) is a frequent consequence of acquired brain injury, especially following right hemisphere damage. Traditionally, unilateral spatial neglect is assessed with cancellation tests such as the Bells test. Recently, a new cancellation test, the Apples test, has been proposed. The present study aims at comparing the accuracy of these two tests in detecting hemispatial neglect, on a sample of 56 right hemisphere stroke patients with a diagnosis of USN. In order to evaluate the agreement between the Apples and Bells tests, Cohen's kappa and McNemar's test were used to assess differences between the two methods of evaluation. Poor agreement and statistically significant differences emerged between the Apples and Bells tests. Overall, the Apples test was significantly more sensitive than the Bells test in detecting USN. Based on these results, the use of the
\end{abstract}

Benedetta Basagni

benedetta.basagni@centrocardinalferrari.it

Antonio De Tanti

antonio.detanti@centrocardinalferrari.it

Alessio Damora

damora@crtspa.it

Laura Abbruzzese

laura.abbruzzese@libero.it

Valentina Varalta

valentina.varalta@univr.it

Gabriella Antonucci

gabriella.antonucci@uniroma1.it

Wai Ling Bickerton

w.bickerton@bham.ac.uk

Nicola Smania

nicola.smania@univr.it
Apples test for peripersonal neglect assessment is therefore highly recommended.

Keywords Neglect · Stroke · Bells test · Apples test · Rehabilitation

\section{Introduction}

According to Heilman [1], unilateral spatial neglect (USN) is commonly defined as the "failure to report, respond, or orient to novel or meaningful stimuli presented to the side opposite a brain lesion, when this failure cannot be attributed to either sensory or motor defects". It often occurs as a consequence of unilateral brain lesion, typically involving the inferior and

Mauro Mancuso

m.mancuso62@gmail.com

1 Cardinal Ferrari Rehabilitation Centre, S. Stefano Institute, via IV Novembre, 21, 43012 Fontanellato, Parma, Italy

2 Tuscany Rehabilitation Clinic, Terranuova Bracciolini, Piazza del Volontariato, 2, 52025 Montevarchi, Arezzo, Italy

3 Neuromotor and Cognitive Rehabilitation Research Centre, Department of Neurological, Biomedical and Movement Sciences, University of Verona, Piazzale Scuro, 10, 37134 Verona, Italy

4 Department of Psychology, Sapienza University of Rome, Via del Marsi, 78, 00185 Rome, Italy

5 IRCCS Fondazione Santa Lucia - Roma, Via Ardeatina 306, 00179 Rome, Italy

6 School of Psychology, University of Birmingham, B15 2TT, Birmingham, UK 
superior parietal lobes, some portions of the frontal lobe [2, 3], and the white matter which links these parietal and prefrontal regions [4-6], especially of the right hemisphere. USN may affect different senses (e.g. vision, hearing, touch, proprioception) and different portions of space (e.g. personal neglect of the body, peripersonal neglect within arm's length and extrapersonal neglect of more distant targets) [7]. Visual neglect is nevertheless generally considered to be a reliable index of outcome, considering the importance of visualperceptive ability in the relationship between subject and the external world.

Symptoms associated with USN may severely impair everyday life activities. For example, patients may consider only the right half of the plate, shave only the right side of their face or forget to look left before crossing the street [8]. Consequently, patients often need continuous care which implies a significant loss of quality of life. Furthermore, several researchers have highlighted that neglect syndrome predicts poorer functional recovery compared to aphasia $[9,10]$. The presence of USN interferes with both motor and cognitive rehabilitations [11]. The outcome can be significantly worse when anosognosia (deficit of self-awareness in which a person with some disability seems unaware of its existence) co-occurs with USN, and the combination of the two reduces the compliance of the patient to the rehabilitative treatment $[12,13]$.

Depending on the study, the incidence of USN varies considerably from 12 to $95 \%$ of right hemisphere stroke patients (for a comprehensive summary, see Robertson and Halligan [14]). Critically for our purpose here, some authors have highlighted that this huge difference in reports may depend upon the assessment tools used in the studies [15]. Due to the severe impact of USN on the recovery in right brain-damaged patients, it is important to assess carefully this syndrome, in order to plan adequate and tailored rehabilitation programmes.

As USN has different manifestations, and there is no shared guideline on the correct way to assess the syndrome, clinicians usually adopt clinical and neuropsychological tools on the basis of their own experience, in order to distinguish patients withUSN from those without-USN. Specific neurological examinations, such as extinction signs or gaze deviation; typical performance at different cancellation tasks; reading, writing, and drawing tests; specific behaviours in daily activities; and TC scan evidence of lesion in the right side of the brain, are taken into account in order to correctly diagnose neglect $[16,17]$.

Peripersonal neglect is often assessed using cancellation tests, where patients are required to tick a series of written stimuli-ignoring distractors scattered among targets (e.g. [18]). Patients with USN typically fail to identify the stimuli distributed in the space located on the opposite side of the damaged hemisphere. Numerous studies have explored the variables that may affect performance in this kind of task, both in healthy and impaired subjects. It has been reported that several factors such as attention demand [19-22], similarity between target and distractor stimuli [23], prominence of the targets [24], number and density of stimuli [25, 26], and the relationship between target and distractor stimuli [27] may affect the performance. In the presence of more than one of these variables, USN becomes particularly evident and specific features of the task seem to affect the sensitivity of the cancellation tasks to detect symptoms of neglect.

Among the different tools normally used to assess the neglect syndrome, the Bells test has a long international history and has become one of the most useful standard tests for diagnosing USN $[28,29]$. The Bells test is a cancellation task where patients select and tick 35 bells from 280 distractors. It is simple to administer and allows clinicians to understand quickly pathological behaviour of patients. Moreover, the Bells test shows a low level of learning effect, so it can be used also for the follow-up evaluations, even when conducted after just few days.

Recently, a new cancellation test has been created: the Apples test [30, 31]. This test enables the detection of both egocentric neglect (patients fail to find stimuli on the left side with respect to egocentric coordinates) and allocentric neglect (patients leave targets with respect to coordinates centered on the object, but independent of the side of space). The Apples test is too a cancellation task, in which patients mark up to 50 apples from 100 distractors, consisting of incomplete apples that can be open on the right or on the left.

The main aim of the present study was to compare the diagnostic accuracy of these two widespread tests often used to assess USN, the Apples and Bells tests, in the assessment of the egocentric aspect of neglect, in patients with right hemisphere stroke.

\section{Methods}

\section{Sample}

The paper is a retrospective study based on the neuropsychological assessment conducted on a consecutive series of right hemisphere stroke patients admitted from September 2014 to December 2016 in three different Italian rehabilitation centres.

The inclusion criteria were patients aged between 18 and 85 years, with right hemisphere stroke (ischemic or hemorrhagic), with a diagnosis of neglect documented through neurological evaluation, in acute or post-acute phase, evidence for CT scan and behavioural signs of neglect. All patients had a silent anamnesis for neurological and psychiatric disturbances. Each patient completed both the Apples and Bells tests.

A total of 56 stroke patients that met these criteria (mean age 65; range 42-85; SD 13) were selected. Nineteen of them had hemorrhagic stroke, whereas 37 had ischemic lesions. 
Table 1 Demographic characteristics of the sample. Centre: 1—Fontanellato (Parma); 2-Montevarchi (Arezzo); 3-Verona. RHD right hemisphere damage, $L H D$ left hemisphere damage

\begin{tabular}{|c|c|c|c|c|c|c|c|}
\hline & \multirow[t]{2}{*}{$N$} & \multicolumn{3}{|c|}{ Aetiology RHD } & \multicolumn{3}{|c|}{ Aetiology LHD } \\
\hline & & Ischemic & Hemorrhagic & Mean age & Ischemic & Hemorrhagic & Mean age \\
\hline Centre 1 & 26 & 16 & 5 & 63 (DS 12) & 2 & 3 & 50 (DS 16) \\
\hline Centre 2 & 32 & 13 & 9 & 68 (DS 14) & 7 & 3 & 71 (DS 13) \\
\hline Centre 3 & 21 & 9 & 4 & 64 (DS 9) & 7 & 1 & 71 (DS 5) \\
\hline
\end{tabular}

In order to assess the pattern of results of patients without visual explorative deficit in the two tests, we also recruited a control group of 23 left hemisphere stroke patients without symptoms of right USN (mean age 66; range 27-87; SD 14.4), 16 with an ischemic lesion and 7 with a hemorrhagic lesion. Table 1 presents the demographic characteristics of both groups (right hemisphere and left hemisphere stroke).

\section{Procedure}

In our sample, all the patients from the three different rehabilitation centres were diagnosed based on a specific neurological examination conducted by expert clinicians, who observed specific neurological signs of neglect. The diagnostic considerations took into account: (1) behavioural presentations including trunk, head and/or gaze deviation through the affected side, specific posture in bed or in the wheelchair, visual or tactile extinction signs, and typical behaviour in daily living activities, such as ignoring the left half of the plate; (2) neuropsychological tests, such as neglecting the stimuli on the left-hand side of the paper or reading the only right-hand side of a newspaper title; and (3) computed tomography (CT) scan showing vascular lesions in the frontal, parietal or temporal areas of the right-hand side of the brain.

All the patients included in this study carried out both the Bells test and the Apples test.

The Bells test consists of 315 stimuli randomly distributed on a A4-sized sheet. The patient was required to identify and tick the scattered 35 bells from 280 distractors (common small figures such as for instance houses, horses etc.). In the sheet, the stimuli are pseudo-randomly organized in seven columns containing five bells each, three on the left-hand side, one in the middle and three on the right. The piece of paper was placed exactly in front of the subject. The patient is allowed a maximum of $5 \mathrm{~min}$ to finish the task. Omissions of targets, cancelled distractors (false alarms) and the difference between the omission on the left three columns and the right three columns are scored. The diagnosis of USN was based on the patient's performance on the final measure which is called "asymmetry score". Bells test is presented in Fig. 1.

Fig. 1 Bells test

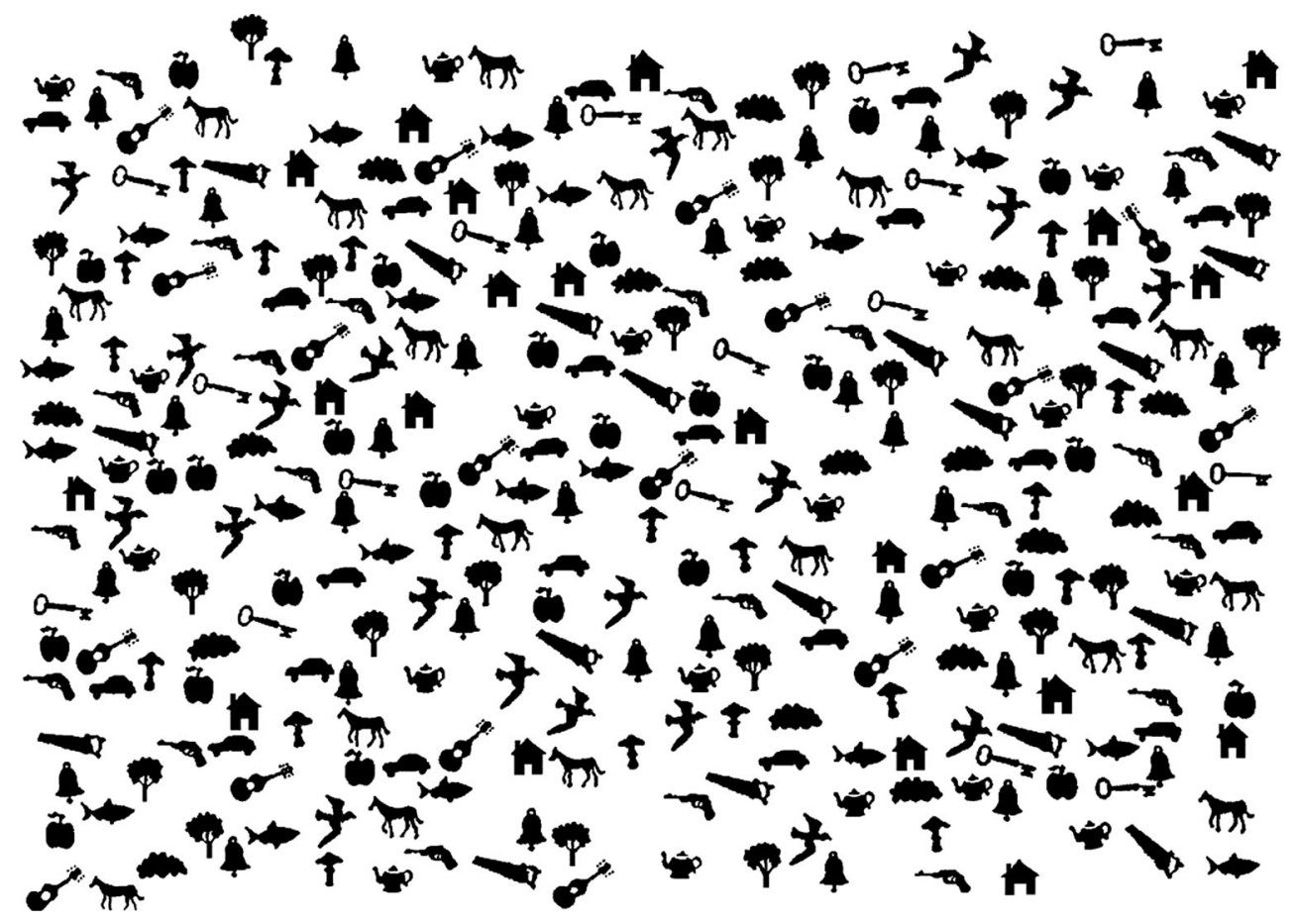


Fig. 2 Apples test

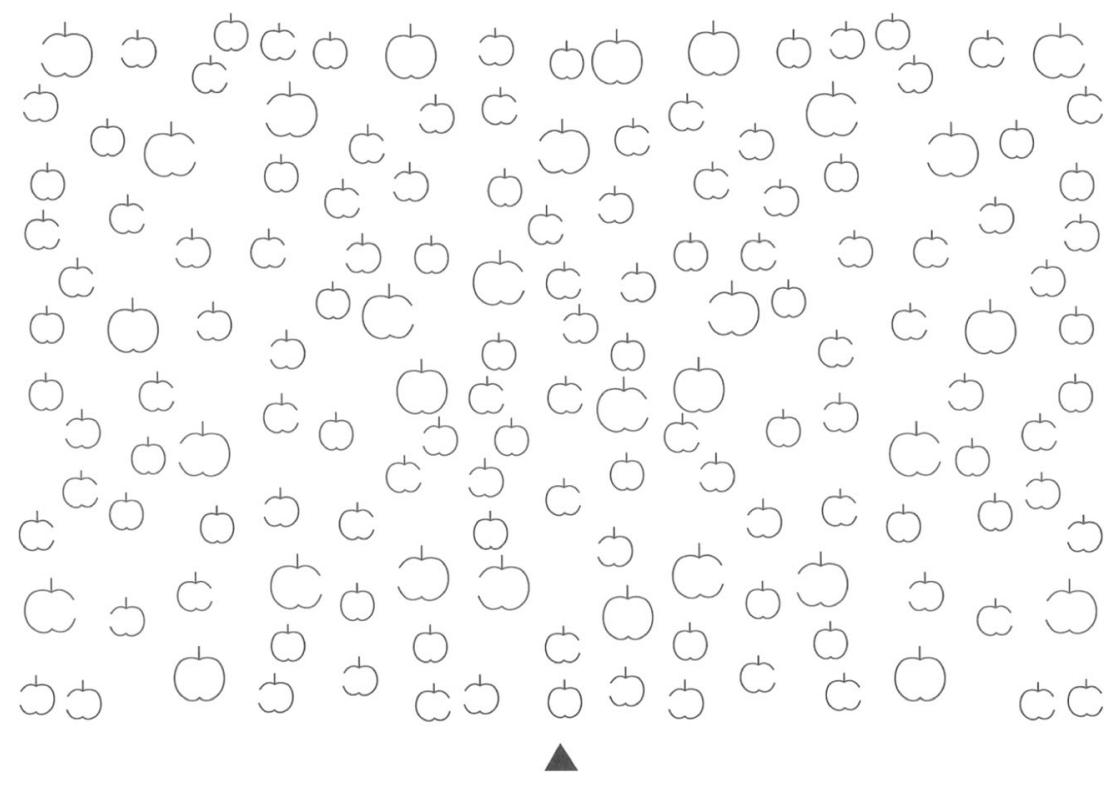

The Apples test is a cancellation task in which outline drawings of 150 apples are shown pseudo-randomly scattered over an A4 sheet presented in a landscape orientation, presented in an upright position. One-third of the apples are complete (50 targets) and two-thirds are open, either on the left- or the right-hand side (distractors). Targets and distractors are evenly distributed; the page is divided into a grid of two rows and five columns. The patient was given $5 \mathrm{~min}$ to cross out all the complete apples. Patients with allocentric neglect tend to ignore the controlesional side of the items, regardless of where they appear with respect to the viewer, while patients with egocentric neglect omit more stimuli presented in the controlesional side of the sheet. Score takes into account the total number of crossed-out targets. The asymmetry score for allocentric neglect corresponds to the difference between the total number of distractor apples cancelled with a left opening (boxes 1 to 4 of the ten boxes of the paper sheet), and the number of distractors cancelled with a right opening (boxes 7 to 10). Positive values indicate left neglect and negative values indicate right neglect. The Apples test is presented in Fig. 2. For this study, we have taken into account only the egocentric score that is the outcome of the Bells test too.

\section{Statistical analyses}

In order to assess the agreement between the Apples and Bells tests, we calculated the Cohen's kappa statistic, $\kappa$, on the total sample. In this statistical test, complete agreement corresponds to $\kappa=1$; no agreement between the two tests corresponds to $\kappa \leq 0$. In addition, McNemar's test of equality of paired proportions was used to find out the difference between the two evaluation methods.
Furthermore, since the sample includes both acute and chronic patients, the same statistical analysis was repeated with sample divided, according to Nijboer [5], into acute phase $(<98$ days from onset) and post-acute phase ( $>98$ days from onset), to verify whether there was a discrepancy in different phases of the illness. Statistical analyses were performed using SPSS Statistics Program software.

\section{Results}

\section{Right hemisphere stroke patients}

In order to assess the agreement between the Apples test and Bells test in detecting the neglect syndrome, we calculated the Cohen's kappa statistic. For this reason, we designed a classification matrix for the binary data (Table 2).

The Cohen's $\kappa$ coefficient $\left(\kappa=\frac{\operatorname{Pr}(a)-\operatorname{Pr}(e)}{1-\operatorname{Pr}(e)}\right)$ ok! showed poor agreement between the Apples test and Bells test $(\kappa=0.13)$. The non-parametric test, McNemar's test, $\left(\kappa=\frac{(b-c) 2}{b+c}\right)$ was statistically significant, showing a difference between the two methods in detecting the neglect syndrome $(p=.00)$. The percentage of true positive (pathological patient testpositive) was statistically higher in the Apples test than in the Bells test. Twenty-nine patients with neglect were detected as positive to the Apples test, but not to the Bells test, revealing higher sensitivity to the Apples test. Six patients were false negative (pathological patient testnegative) for both tests. The percentage of agreement was $48 \%[(21+6) / 56]$ 
Table 2 Classification matrix of the performance of the sample to the Apples test and Bells test

\begin{tabular}{lllll}
\hline & \multicolumn{5}{c}{ Bells test } \\
\hline \multirow{3}{*}{ Apples test } & & + & - & \\
& + & 21 & 29 & 50 \\
& - & 0 & 6 & 6 \\
& & 21 & 35 & 56 \\
\hline
\end{tabular}

\section{Right hemisphere stroke patients: acute versus post-acute patients}

Twenty-nine of the patients were in an acute phase, with a mean time from the onset of 40 days (SD 24), and 27 patients were in the post-acute phase, with a mean time from the onset of 596 days (SD 855). No significant differences were found concerning the average age (group 1: mean age 64.8; group 2: mean age $65.2 ; t<1$ ). We then created the two classification matrices both for acute-phase and post-acute-phase patients (see Tables 3 and 4).

Cohen's analysis showed that agreement between the Apples test and the Bells test was higher in the acute sample $(\kappa=0.17)$ compared to the post-acute sample $(\kappa=0.09)$. In both samples, however, the difference between the two methods of evaluation in detecting neglect syndrome was statistically significant (both $p=.00$ ), with a percentage of true positive (pathological patient testpositive) statistically higher for the Apples test, compared to the Bells test. Fourteen patients in the acute sample and 15 in the post-acute sample were found to be impaired to the Apples test, but not to the Bells test. The percentage of agreement for acute patients was $51 \%[(11+4) / 29]$, while for post-acute patients was $44 \%[(10+2) / 27]$.

\section{Left hemisphere stroke patients}

Of the 23 left brain-damaged patients, no one presents a significant asymmetry score both for the Apples and Bells tests. Pathological scores were nevertheless registered in total omissions, both for the Apples test $(6 / 23,26 \%)$ and Bells test $(10 / 23,43 \%)$.

Table 3 Classification matrix of performance to the Apples test and Bells test in the acute-phase sample

\begin{tabular}{lllll}
\hline & \multicolumn{5}{c}{ Bells test } \\
\hline \multirow{3}{*}{ Apples test } & & + & - & \\
& + & 11 & 14 & 25 \\
& - & 0 & 4 & 4 \\
& & 11 & 18 & 29 \\
\hline
\end{tabular}

Table 4 Classification matrix of performance to the Apples test and Bells test in the post-acute-phase sample

\begin{tabular}{lllll}
\hline & \multicolumn{5}{c}{ Bells test } \\
\hline \multirow{3}{*}{ Apples test } & & + & - & \\
& + & 10 & 15 & 25 \\
& - & 0 & 2 & 2 \\
& & 10 & 17 & 27 \\
\hline
\end{tabular}

\section{Discussion and conclusion}

The aim of this study was to compare the accuracy of two widely used cancellation tests (the Apples test and the Bells test) which detect peripersonal USN, using a sample of right hemisphere stroke patients.

Our results show that the Apples test appears to be more sensitive than the Bells test. Out of the 56 patients with USN, the Apples test detected 50 patients (89.2\%), while the Bells test detected only 21 patients $(37.5 \%)$ among patients with right brain lesion.

We also studied a sample with left brain lesion without symptoms of USN. As expected, neither the Apples test nor the Bells test presented significant asymmetry scores. Both tools detected nevertheless in some cases scattered omission errors, as index of attention disorder. These findings provide useful information for clinical neuropsychological assessment.

In our right hemisphere stroke sample, we included both acute and post-acute patients, since the timing from onset did not represent a significant variable. Indeed, statistical analysis comparing these two groups highlighted the absence of significant differences between them. The discrepancy between the Apples test and the Bells test in detecting patients affected by neglect syndrome seems therefore to be unrelated to distance from the onset time. However, the discrepancy appears to be more likely to be dependent on the characteristics of the tests themselves. Indeed, both tasks show several differences. First, they are different in the density of stimuli. The Bells test presents a total of 315 shapes, while the Apples test only 150 , both on a sheet of equal dimensions: the density of stimuli for the Bells test is therefore greater. Secondly, the ratio of targets to distractors is $35 / 315$ ( 1 over 9 ) in the Bells test and 50/150 (1 over 3 ) in the Apples test. Thirdly, they are different in targets/distractors similarity. In the Apples test, the distractors are more similar to the target stimuli than in the Bells test, and the prominences of the target are therefore lower. The high degree of similarity between targets and distractors seems to have a significant weight with respect to density, number and ratio of target to distractor. This makes the Apples test certainly harder and more demanding in terms of attention.

Our study presents nevertheless several limitations. The sample is composed of narrowly defined right hemisphere brain-damaged patients. Future investigations should include 
a perspective multicentric study with a broad sample of patients, including also left lesions. Moreover, the characteristics of the damage (e.g. severity) should be further investigated, as well as sociodemographic property (e.g. age), to find correlations with the accuracy of the two tests.

In conclusion, our preliminary results suggest that compared to the Bells test, the Apples test is preferred for peripersonal neglect assessment, where the detection of the syndrome is crucial to inform treatment and rehabilitation.

\section{Compliance with ethical standards}

Ethical statement For this type of study (retrospective), formal consent is not required.

Conflict of interest The authors declare that they have no conflict of interest.

\section{References}

1. Heilman KM, Watson RT, Valenstein E (1993) Neglect and related disorders. In: Heilman KM, Valenstein E (eds) Clinical neuropsychology. Oxford University Press, NewYork, pp 279-336

2. Husain M, Mattingley JB, Rorden C et al (2000) Distinguishing sensory and motor biases in parietal and frontal neglect. Brain 123:1643-1659

3. Ringman JM, Saver JL, Woolson RF et al (2004) Frequency, risk factors, anatomy, and course of unilateral neglect in an acute stroke cohort. Neurology 63:468-474

4. Bartolomeo P, Thiebaut De Schotten M, Doricchi F (2007) Left unilateral neglect as a disconnection syndrome. Cereb Cortex 17: 2479-2490

5. Nijboer TC, Kollen BJ, Kwakkel G (2013) Time course of visuospatial neglect early after stroke: a longitudinal cohort study. Cortex 49:2021-2027

6. Karnath HO (2001) New insights into the functions of the superior temporal cortex. Nat Rev Neurosci 2:568-576

7. Corbetta M (2014) Hemispatialneglect: clinic, pathogenesis, and treatment. Semin Neurol 34:514

8. Nijboer TC, Kollen BJ, Kwakkel G (2014) The impact of recovery of visuo-spatial neglect on motor recovery of the upper paretic limb after stroke. PLoSOne 9(6):e100584

9. Paolucci S, Antonucci G, Gialloreti LE et al (1996) Predicting stroke in patient rehabilitation outcome: the prominent role of neuropsychological disorders. Eur Neurol 36:385-390

10. Jehkonen M, Ahonen JP, Dastidar P et al (2000) Visual neglect as a predictor of functional outcome one year after stroke. Acta Neurol Scand 101:195-201

11. Mutai H, Furukawa T, Araki K et al (2012) Factors associated with functional recovery and home discharge in stroke patients admitted to a convalescent rehabilitation ward. Geriatr Gerontol Int 12:215-222

12. Appelros P, Karlsson GM et al (2003) Prognosis for patients with neglect and anosognosia with special reference to cognitive impairment. J Rehabil Med 35:254-258

13. Gialanella B, Monguzzi V et al (2005) Functional recovery after hemiplegia in patients with neglect: the rehabilitative role of anosognosia. Stroke 36:2687-2690

14. Robertson IH, Halligan PW (1999) Spatial neglect: a clinical handbook for diagnosis and treatment. Psychology Press, Hove

15. Bowen A, McKenna K, Tallis RC (1999) Reasons for variability in the reported rate of occurrence of unilateral spatial neglect after stroke. Stroke 30(6):1196-1202

16. Bowen A, Lincoln NB, \& Dewey M (2007) Cognitive rehabilitation for spatial neglect following stroke. Cochrane Database Syst Rev 2

17. Wilson BA, Cockburn J, Halligan PW (1987) Behavioural inattention test: manual. Thames Valley Test Company, Fareham

18. Albert ML (1973) A simple test of visual neglect. Neurology 23(6): 658-664

19. Kinsbourne M (1987) Mechanisms of unilateral neglect. In: Jeannerod M (ed) Neurophysiological and neuropsychological aspects of spatial neglect. Elsevier, Amsterdam, pp 69-86

20. Behrmann M, Ebert P, Black SE (2004) Hemispatial neglect and visual search: a large scale analysis. Cortex 40(2):247-263

21. Bonato M (2012) Neglect and extinction depend greatly on task demands: a review. Front Hum Neurosci 6:195

22. Ricci R, Salatino A, Garbarini F et al (2016) Effects of attentional and cognitive variables on unilateral spatial neglect. Neuropsychologia 92:158-166

23. Riddoch MJ, Humphreys GW (1987) Perceptual and action systems in unilateral visual neglect. Adv Psychol 45:151-181

24. Weintraub S, Mesulam MM (1988) Visual hemispatial inattention: stimulus parameters and exploratory strategies. J Neurol Neurosurg Psychiatry 51(12):1481-1488

25. Mark VW, Kooistra CA, Heilman KM (1988) Hemispatial neglect affected by non neglected stimuli. Neurology 38(8):1207-1207

26. Eglin M, Robertson LC, Knight RT (1989) Visual search performance in the neglect syndrome. J Cogn Neurosci 1(4):372-385

27. Kaplan RF, Verfaellie M, Meadows ME et al (1991) Changing attentional demands in left hemispatial neglect. Arch Neurol 48(12):1263-1266

28. Gauthier L, Dehaut F, Joanette Y (1989) The bells test: a quantitative test for visual neglect. Int J Clin Neuropsychol 11:49-54

29. Oliveira De CR, Luara De FC et al (2016) Use of Bells test in the evaluation of the hemineglect post unilateral stroke. J Neurol Neurosci 7:1-8

30. Bickerton WL, Samson D et al (2011) Separating forms of neglect using the Apples test: validation and functional prediction in chronic and acute stroke. Neuropsychology 25(5):567

31. Mancuso M, Rosadoni S, Capitani D et al (2015) Italian standardization of the Apples Cancellation Test. J Neurol Sci 36(7): $1233-1240$ 